\title{
Cardiovascular magnetic resonance in an adult human population: serial observations from the multi-ethnic study of atherosclerosis
}

\author{
Kihei Yoneyama ${ }^{1,4}$, Bharath A. Venkatesh ${ }^{1}$, David A. Bluemke², Robyn L. McClelland ${ }^{3}$ and João A.C. Lima ${ }^{1,5^{*}}$
}

\begin{abstract}
The Multi-Ethnic Study of Atherosclerosis (MESA) is the first large-scale multi-ethnic population study in the U.S. to use advanced cardiovascular magnetic resonance (CMR) imaging. MESA participants were free of cardiovascular disease at baseline between 2000 and 2002, and were followed up between 2009 and 2011 with repeated CMR examinations as part of MESA. CMR allows the clinician to visualize and accurately quantify volume and dimensions of all four cardiac chambers; measure systolic and diastolic ventricular function; assess myocardial fibrosis; assess vessel lumen size, vessel wall morphology, and vessel stiffness. CMR has a number of advantages over other imaging modalities such as echocardiography, computed tomography, and invasive angiography, and has been proposed as a diagnostic strategy for high-risk populations. MESA has been extensively evaluating CMR imaging biomarkers, as markers of subclinical disease, in the last 15 years for low-risk populations. On a more practical level, some of the imaging biomarkers developed and studied are translatable to at-risk populations. In this review, we discuss the progression of subclinical cardiovascular disease and the mechanisms responsible for the transition to symptomatic clinical outcomes based on our findings from MESA.
\end{abstract}

Keywords: Ageing, Fibrosis, Heart failure, Cardiovascular disease, Torsion

\section{Background}

The Multi-Ethnic Study of Atherosclerosis (MESA) is a prospective study sponsored by the National Heart, Lung, and Blood Institute (NHLBI) of the National Institutes of Health. The major objective of MESA was to evaluate the mechanisms that underlie the development and progression of subclinical cardiovascular disease (CVD) among asymptomatic individuals in the general population. MESA was primarily designed to study the progression of subclinical CVD and the mechanisms responsible for the transition to symptomatic clinical outcomes [1]. In brief, between July 2000 and August 2002, 6814 men and women, 45 to 84 years of age-who identified

\footnotetext{
*Correspondence: jlima@jhmi.edu

${ }^{1}$ Department of Cardiology, Johns Hopkins University, Baltimore, MD, USA

${ }_{5}^{5}$ professor of Medicine, Radiology and Epidemiology, Johns Hopkins Hospital, Johns Hopkins University, Blalock 524D1, 600 North Wolfe Street, Baltimore, MD 21287, USA

Full list of author information is available at the end of the article
}

themselves as Caucasian, African American, Hispanic, or Chinese, and who were free of clinically apparent CVD were recruited. Cardiovascular magnetic resonance imaging (CMR) was performed on 5004 participants as part of the baseline examination and 3015 underwent a followup CMR between 2010 and 2012 (year-10).

CMR is a noninvasive medical test that creates detailed pictures of the beating heart and vessels to look at their structure and function. In MESA, CMR was used to accurately quantify volume and dimensions of all four cardiac chambers, systolic and diastolic ventricular and atrial function, assess extent of replacement and interstitial fibrosis, and measure aortic structure and function, to evaluate patterns of cardiac structural remodeling and the prevalence of myocardial fibrosis across the life course, identify the myocardial mechanical consequences of remodeling, explore ventricular-arterial coupling and the role of arterial stiffness in CVD, and to study the prognostic importance of CMR biomarkers. 
In this review, we discuss the progression of subclinical CVD and the mechanisms responsible for the transition to symptomatic clinical outcomes based on our findings from MESA.

\section{Left ventricular (LV) remodeling across the life course}

The observed variations in the structure of the left ventricle might be attributable to differences in demographic variables (e.g., age, gender, and race), traditional risk factors (e.g., LV overload by blood pressure or atherosclerosis), other medical conditions, socioeconomic status, and genetic influences in general populations. In MESA, LV wall thickness by CMR was associated with gender, race, body size, and LV function [2]. Chinese participants had lower volumes and mass than other ethnic groups among normal participants without known CVD risk factors [3]. LV volumes and mass were significantly greater for men than women, and, more spherical shape of LV was seen in women as compared to men [4-6].

The average age of populations around the globe is increasing; as a result, an increasing number of older individuals become afflicted by CVD [7]. While age is one of the most powerful risk factors for CVD, it is important to understand the LV adaptation mechanisms that function against the altered cardiovascular system during aging in human adult life. The findings of MESA CMR related to aging are summarized in Fig. 1. The MESA serial longitudinal study confirmed that a pattern of LV remodeling across the life course is concentric remodeling with progressively reduced LV volumes resulting in an increased LV mass-to-volume ratio. Specifically, we see a significant age-related longitudinal increase in LV mass in men and a slight longitudinal decrease in women over 9 years follow-up; additionally, longitudinal decreases in LV end-diastolic volume and stroke volume were seen in both men and women, leading to significant increases in adverse LV concentric remodeling (increased massto-volume ratio) in both men and women [8]. The LV ejection fraction (LVEF), however, was maintained with age due to a progressive decline in LV end-diastolic volume (Fig. 1) [5].

Aging prolongs exposure to CVD risk factors, therefore, understanding the influences of adverse LV remodeling by risk factors in the general population is important. In cross-sectional studies, CVD risk factors were associated with LV concentric remodeling (i.e., increased blood pressure, body mass index, body fat, insulin resistance, and presence of hypertension and diabetes), even though variants of LV remodeling patterns were observed with

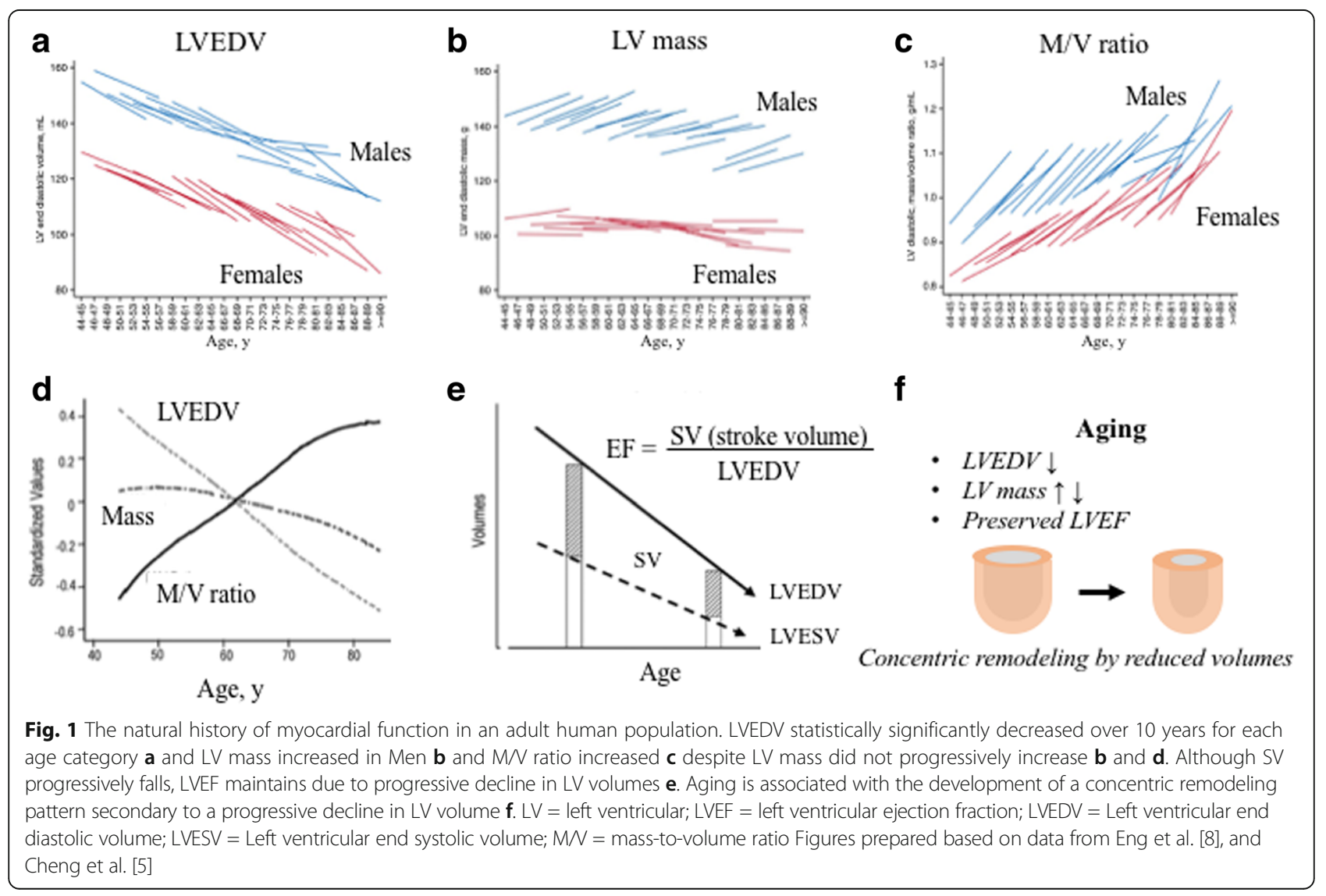


each CVD risk factor (i.e, hypertensive participants had concentric remodeling with greater LV volumes, whereas diabetic participants had concentric remodeling with lower volumes) [4, 6, 9-12]. In addition, a higher anklebrachial index, traffic exposure, and genetic variation were associated with higher LV mass [13-15]. Higher physical activity was associated with higher LV mass but not with concentric remodeling, and higher activity was associated with lower heart rate [16]. MESA longitudinal studies confirmed some of the findings of the cross-sectional studies by showing that changes in CVD risk factors are associated with changes in LV remodeling, e.g., weight loss, reduced blood pressure, and reduced heart rate were associated with a smaller increase in age-related concentric remodeling $[11,12]$.

\section{LV remodeling and cardiac events}

Progressive LV remodeling may identify individuals at high risk for cardiac events in the general population. For example, in contrast to the natural history of concentric remodeling, the risk of incident CVD is greater for those individuals who develop concentric remodeling at a younger age (Fig. 2a). This pattern of ventricular remodeling appears to confer significant CVD risk, especially when present earlier in life [5].

Heart failure (HF) is an important contributor to the burden and cost of national healthcare expenditures, and with the aging of worldwide populations, the estimated prevalence and cost of care for HF will increase markedly for all sex and racial/ethnic subgroups [7, 17]. The pattern of hypertrophy is related to pressure overload leading to concentric hypertrophy and volume overload leading to eccentric hypertrophy.

The Framingham study has suggested that early identification of LV hypertrophy by electrocardiogram
(ECG) or echocardiography may provide an opportunity to prevent the development of cardiac events, however, the MESA CMR study found that ECG had low sensitivity (10\% to $26 \%$ ) for detecting CMR-defined hypertrophy [18], and that CMR is highly accurate using 3-dimensional ventricular size and shape compared to conventional echocardiography [19]. The predictive value of CMR for heart failure (HF) events exceeded that of coronary artery calcium (CAC) and carotid intima-medial thickness (IMT) for both men and women [20].

In MESA, higher LV mass at baseline ( $\geq 95$ th percentile compared to the reference group of the $<50 \%$ percentile) is strongly associated with incident $\mathrm{HF}$ with a hazard ratio of 8.6 (95\% confidence interval: 3.9 to 19.9) (Fig. 2b) [21]. The addition of N-terminal pro b-type natriuretic peptide (NT-pro BNP) with LV mass produced the largest increase in the c-statistic over the basic traditional risk assessment for predicting HF (Fig. 2c) [22]. Extreme LV sphericity was associated with higher incidence of $\mathrm{HF}$ and atrial fibrillation [23]. The strengths of MESA include high-quality assessments of multiple risk factors for $\mathrm{HF}$ including traditional risk factors and biomarkers, which are responsible for the high predictive value of the MESA HF score [22].

\section{LV replacement fibrosis in the general population}

Late gadolinium-enhancement imaging (LGE) can be used to detect replacement or focal myocardial fibrosis (scar) with a high degree of accuracy $[24,25]$. CMR LGE represents end-organ damage and worse cardiac prognosis among patients with cardiomyopathy [26, 27]; identification of LGE might significantly improve risk stratification in the general population.

From a community-based cohort in Iceland with a median age of 76 years, the prevalence of unrecognized

a $\mathrm{M} / \mathrm{V}$ ratio and cardiovascular disease $\mathbf{b}$ LV mass and incident heart failure $\mathbf{C}$ Heart failure prediction from MESA
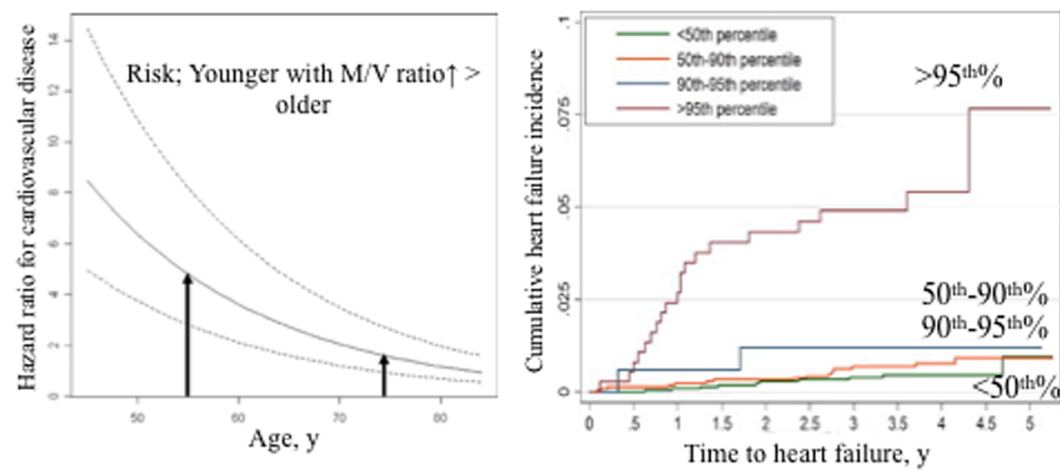

\begin{tabular}{lcc}
\hline \multicolumn{1}{c}{ Models } & C-statstic & $\begin{array}{c}\text { NRI } \\
\text { (p value) }\end{array}$ \\
\hline Traditional risk & 0.80 & \\
(Risk)+LV mass & 0.85 & $\begin{array}{c}0.25 \\
(<0.001)\end{array}$ \\
$\begin{array}{lcc}\text { (Risk+LV mass)+logNT- } \\
\text { proBNP }\end{array}$ & 0.89 & $\begin{array}{c}0.15 \\
(0.018)\end{array}$
\end{tabular}

$\mathrm{NRI}=$ net reclassification improvement, NTproBNP $=\mathrm{N}$-terminal pro-B-type natriuretic peptide.

Fig. 2 Left ventricular remodeling and incident cardiac events in MESA. The risk of incident cardiovascular disease is greater for those individuals who develop the concentric remodeling at a younger compared with older age (black arrows, a). Higher LV mass (hypertrophy) is associated with incident heart failure $\mathbf{b}$. Additional LV mass and log NT-pro BNP produces the largest increased in c-statistic and improves the NRI beyond traditional risk factors c. Figures prepared based on data from Cheng et al. [5], Bluemke et al. [21], and Chahal et al. [22]. Abbreviations as in Fig. 1 
myocardial infarction (MI) detected by CMR with LGE was $17 \%$ (157/936), and was associated with increased mortality similar to recognized MI, compared to individuals with no MI [28]. In MESA, of 1840 participants with a mean age of 68 years, $7.9 \%$ had myocardial scars defined by focal LGE, of which $78 \%(114 / 146)$ were undetected by ECG or by clinical adjudication [29]. The following individual risk factors were associated with higher odds of myocardial scar: age, male sex, body mass index, current smoking, and hypertension at baseline in 2000 to 2002 [29]. Importantly, CAC score by CT at baseline was associated with a typical ischemic scar pattern in 2009 to 2011 [29]. These suggest that unrecognized MI appears to represent a phenotype of coronary heart disease related to atherosclerosis risk factors.

From a clinical longitudinal study of patients with idiopathic, dilated cardiomyopathy (non-ischemic) receiving optimal medical therapy, LV systolic function decreased during a 24-month follow-up in patients with myocardial scar at baseline, while reverse remodeling was observed in patients without myocardial scar [30]. MESA reported results similar to that in a high-risk population, namely that asymptomatic individuals with prevalent LGE had an enlarged left ventricle (concentric hypertrophy in women and LV dilatation in men) with progressively declining LVEF; conversely, individuals without myocardial scar maintained LV function [31]. Therefore, the presence of replacement fibrosis was associated with changing LV volumes and reduced function over time even in individuals in lower-risk populations, and who may be at risk to develop asymptomatic HF regardless of whether fibrosis was ischemic or atypical.

\section{LV interstitial fibrosis in a general population}

Although LGE CMR allows for the assessment of replacement myocardial fibrosis, it is limited in the evaluation of diffuse interstitial fibrosis. T1 myocardial mapping-a fairly new technique that can be used to identify the exact T1 value of tissue-enables direct myocardial signal quantification, characterization of myocardial tissue composition and assessment of interstitial fibrosis, and correlates with interstitial fibrosis as assessed by means of invasive biopsy $[24,25]$.

Our study, using contrast T1 found the following two main results: First, the age-related remodeling pattern of left ventricule-which is a progressive decline in LV volumes leading to concentric remodeling-is seen with progressive LV diffuse fibrosis. In other words, persions with either greater LV mass or without concentric remodeling are likely to have less interstitial fibrosis on average [31, 32]. Cardiac aging, characterized by shrinking LV volume with diffuse interstitial fibrosis, can support the pathological finding of an age-related increase in myocyte deaths accompanied with reductions in coronary vasculature through the cardiac life course [31, 33]. Second, hypertension contributed to interstitial fibrosis [31, 32]. This also supports the pathological findings of increases in the extracellular matrix by accumulation of perivascular fibrosis and fibrosis of the endomysium and perimysium in hypertensive heart disease [34].

In MESA, both replacement and interstitial fibrosis are important determinants of LV function, as shown in Fig. 3. Interestingly, men had a linear decline in LV systolic function with interstitial fibrosis, whereas women had a progressive decline in LV diastolic (not systolic) function with fibrosis [31,35]. Perhaps restrictive filling with eventual diastolic dysfunction-due to progressive diffuse myocardial fibrosis with fewer myocytes (related to aging) - could progress to a stiffer left ventricule. In addition, men had progressively increased interstitial fibrosis with burden of CVD risk factors, while women were likely to have more interstitial fibrosis than men independent of CVD risk factors [32, 36]. Of note, these results concur with a previous epidemiology study finding that diastolic HF is more common in elderly men and women than in younger patients [37].

\section{Cardiac deformation imaging using tagged CMR}

CMR tissue tagging provides precise quantification of incipient myocardial dysfunction through the assessment of myocardial strain and torsion, and has been used as the reference standard to validate two-dimensional speckle tracking echocardiography.

In the MESA population, reduced regional peak systolic circumferential strain was associated with impaired regional myocardial perfusion in participants who did not have relevant coronary artery stenosis (Fig. 4a) [38]. This suggests that impaired myocardial shortening enhances the ability to identify early subendocardial ischemia and has a potential role in risk stratification beyond traditional LVEF. For example, worse circumferential myocardial strain has been observed to be associated with LV hypertrophy, concentric remodeling, subclinical atherosclerosis, older age, male sex, hypertension, resting higher heart rate, smoking, and metabolic syndrome [5, 6, 39-44], and has been shown to have incremental predictive value for HF events beyond the traditional LVEF (Fig. 4b) [45]. Diastolic function from circumferential strain curves showed a powerful independent ability for the prediction of HF and atrial fibrillation over an 8-year follow-up period [46]. In addition, tagged CMR provides further insight into the depth of the cardiac pump mechanism in humans. For example, it has been noted that perhaps the discrepancy between the age-associated increase in LVEF and the decline in circumferential shortening can be explained by the 

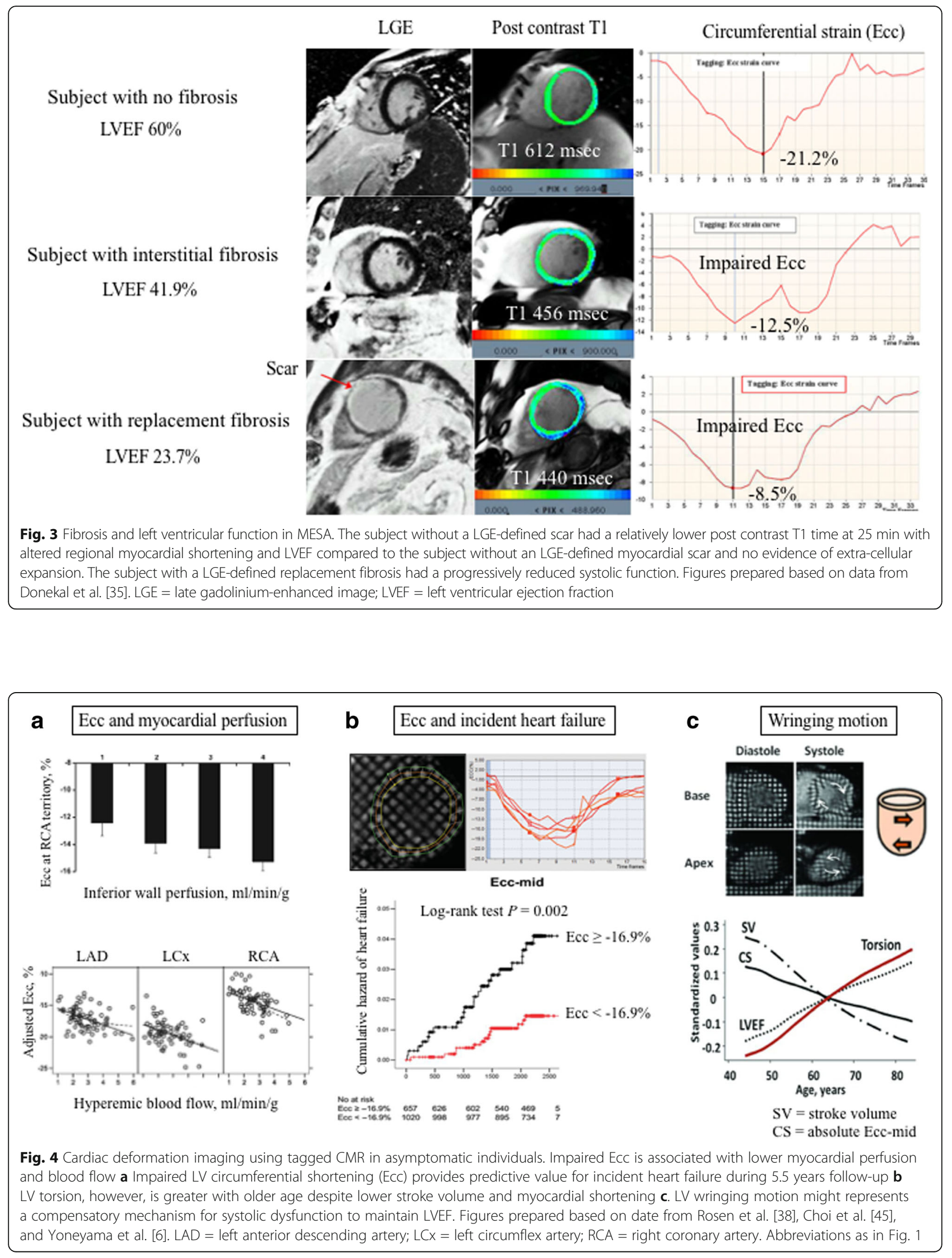
enhanced wringing motion of the left ventricule assessed by tagged CMR (Fig. 4c) [6], which represents a human compensatory mechanism to maintain LVEF in agerelated concentric remodeling.

Finally, progression of symptomatic events from asymptomatic individuals is summarized in Fig. 5.

\section{A novel CMR marker for LV trabeculation}

Clinically, LV noncompaction is a myocardial disorder characterized by excessive LV trabeculae. Although a high degree of noncompacted (trabeculated) myocardium in relationship to compact myocardium (trabeculated to compact myocardium $[\mathrm{T} / \mathrm{M}]$ ratio $>2.3$ ) has been associated with a diagnosis of LV noncompaction, MESA found that $43 \%$ of subjects without cardiac disease or hypertension had a $\mathrm{T} / \mathrm{M}$ ratio $>2.3$ in at least 1 myocardial segment [47]. Using fractal dimension, which is an automated assessment for trabecular quantification that is relatively insensitive to compacted myocardium wall thickness, the LV was more trabeculated in African-American and Hispanic participants than white participants, and smoothest in Chinese-American participants [48]. MESA also confirmed in a longitudinal observation study that a greater extent of LV trabeculation was not associated with an absolute decline in LVEF during the approximately 10 years of the MESA study. Our findings suggest that in subjects with a low pre-test probability for cardiomyopathy and marked trabeculation, greater trabeculation is not related to ejection fraction in asymptomatic subjects [49]. However, the relationship of high degrees of trabeculations to regional function and other remodeling mechanisms has not yet been determined [50].

\section{Right ventricle in a general population}

CMR is the preferred method for evaluating right ventricular structure and function due to its crescent-shape in cross-sectional images. In MESA, older age was associated with lower RV (right ventricular) mass and higher RV ejection fraction; men had greater RV mass and larger RV volumes than women, but had lower RV ejection fraction; African-Americans had lower RV mass than whites, whereas Hispanics had higher RV mass [51]. Traffic exposure was associated with higher RV mass, i.e., participants who lived in the same neighborhood for several years had the strongest associations

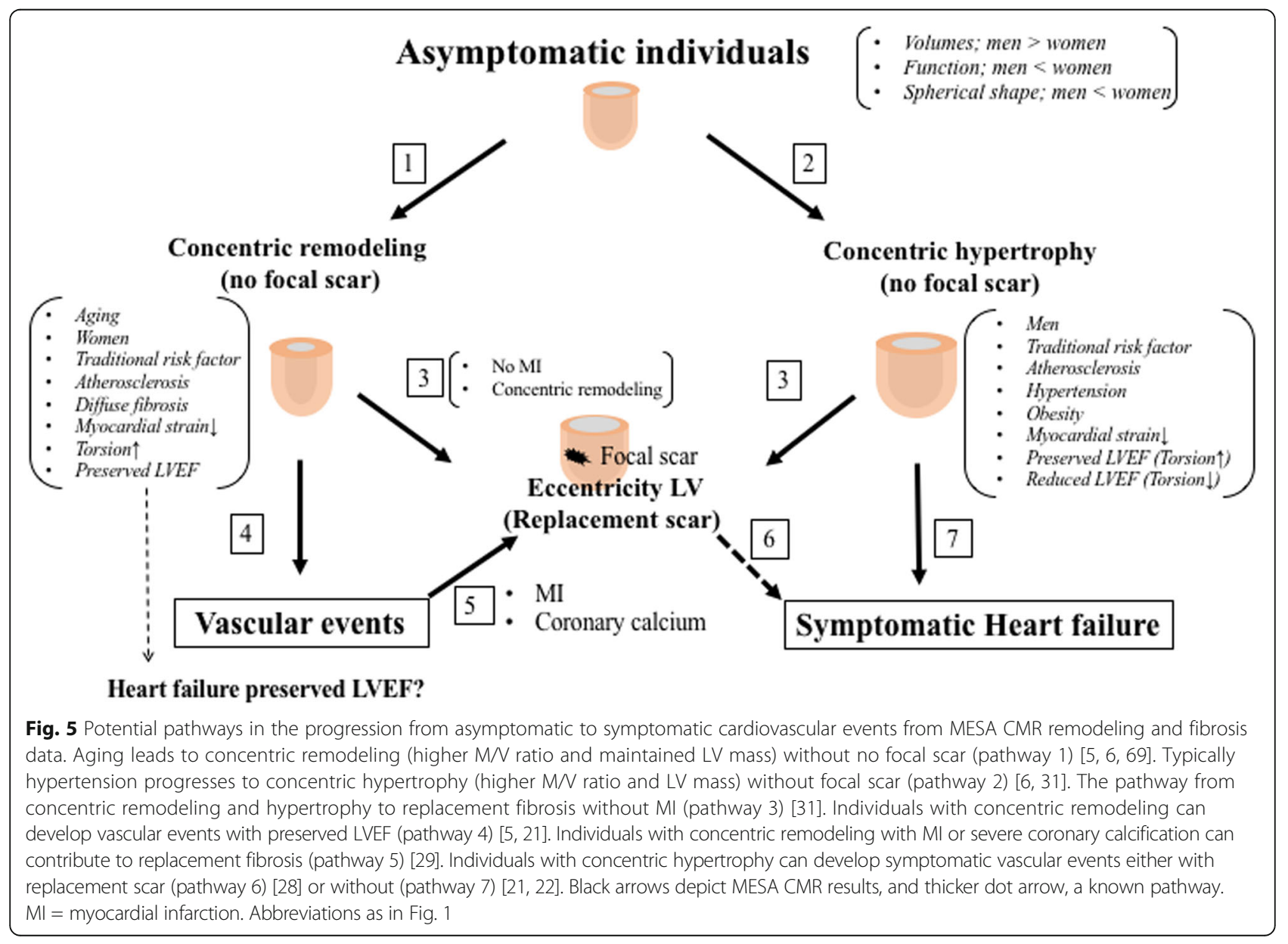


between nitrogen dioxide and RV mass [52]. Participants with RV hypertrophy had a significant increase in the risk of HF or death that was independent of LV mass, and had a stronger association with the outcome in the setting of lower LV mass [53].

\section{Left atrial (LA) measurements by tissue-tracking CMR in asymptomatic individuals}

In normal sinus rhythm, LA performance is characterized by a complex of three basic functions: reservoir function (collection of pulmonary venous flow during LV systole), conduit function (passage of blood to the LV during early diastole), and active booster pump function (augments LV filling during LV late diastole) [54, 55]. Hoit's review of clinical studies examining LA size and function found that LA ejection fraction, which is volume assessment, is a promising measure for predicting CVD in a patient population [56].

Tissue-tracking on LA wall from cine CMR can provide an alternative and additional assessment of risk

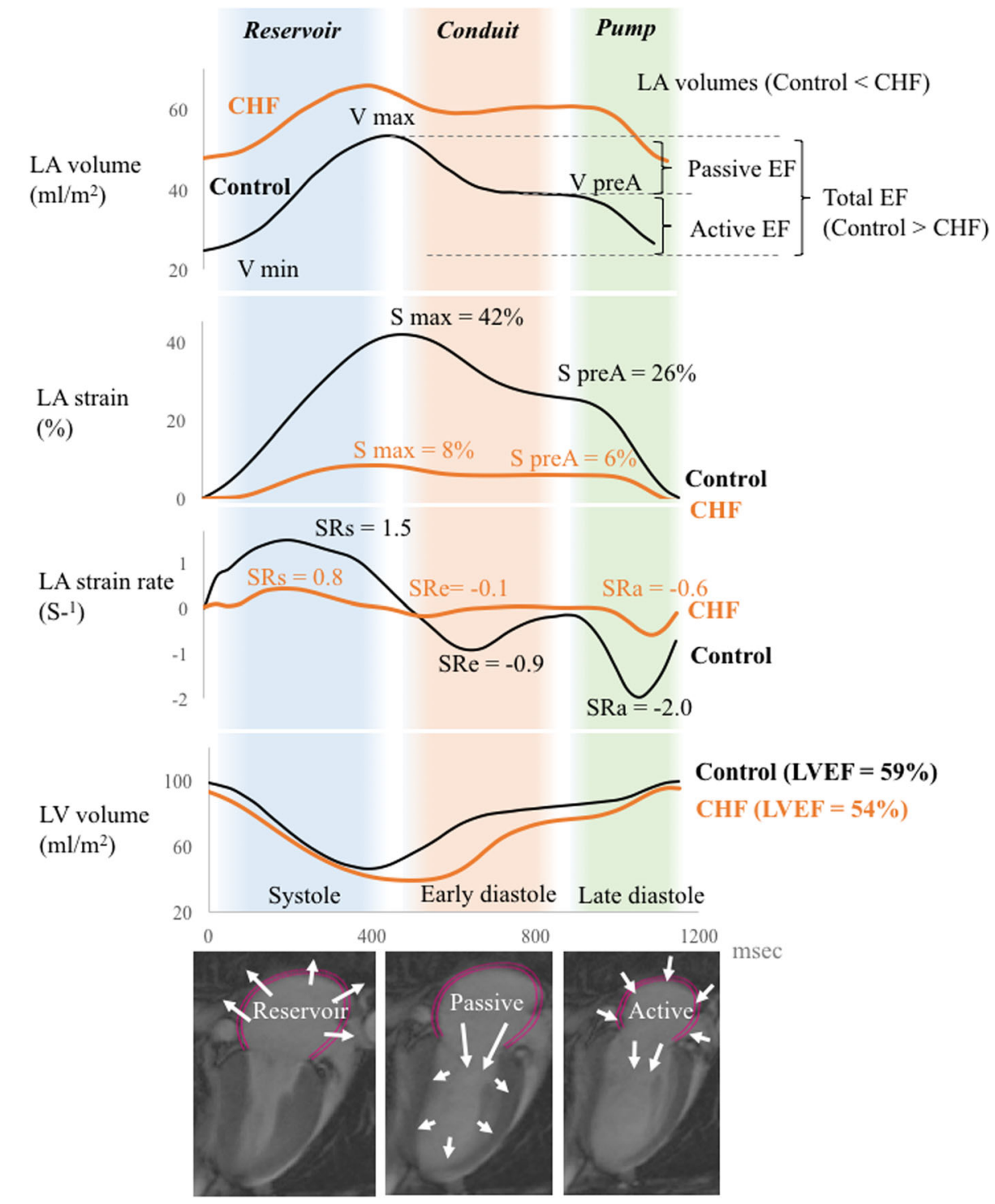

Fig. 6 Left atrial measurements by tissue-tracking CMR in asymptomatic individuals. LA volume, LA strain, LA strain rate, and LV volume in a asymptomatic participant free of cardiovascular daises as a control (block line) and a MESA participant who developed CHF (orange line). A case of CHF had a relatively greater LA volumes with impaired LA strains ( $S$ max and S preA) and LA strain rates (SRs, SRe, and SRa) than a control. Volumes are index to body surface area. LA function was analyzed by using a tissue-tracking method with semiauto mated software (multimodality tissue tracking [MTT] version 5.0; Toshiba, Tochigi, Japan). CHF; congestive heart failure; LA = left atrial; LV = left ventricular; $S$ max = maximum strain; S preA = pre atrial contraction strain; $\mathrm{SRa}=$ strain rate at atrial contraction; $\mathrm{SRe}=$ strain rate at $\mathrm{LV}$ early diastole; $\mathrm{SRs}=$ maximum strain rate, $\mathrm{V}$ max $=$ maximum indexed volume; $\vee \mathrm{min}=$ minimum indexed volume; $\mathrm{V}$ pre $\mathrm{A}=$ pre-atrial contraction indexed volume 
stratification or diastolic function beyond LA volume assessment. Data from a clinical study using this technique on 169 patients with a history of atrial fibrillation indicated that lower LA strain and strain rate during LV diastole (impaired LA reservoir function) is significantly associated with a prior history of stroke, and that the incremental value of LA strain for diagnosis of stroke is further increased by adding LA global strain to LA volume [55]. Similarly, in the MESA population, asymptomatic individuals who developed HF had a lower LA strain during LV diastole (impaired LA reservoir function) and a higher minimum LA volume (impaired pump function and volume) than those without HF events. The deteriorations in LA performance appeared to robustly predict $\mathrm{HF}$ events independent of LV mass and NT-pro BNP [57].

Our study also reported the following: first, a presence of replacement scar on LV was associated with all three basic LA performance functions including an impaired reservoir function (lower maximum LA strain and strain rate), a conduit function (lower absolute strain rate), and a pump function (lower absolute strain rate), but was not significantly associated with total LAEF. Second, LV diffuse myocardial fibrosis assessed by contrast T1 was associated with an impaired reservoir function (lower LA strain) and a pump function (lower LA strain rate) but was not significantly associated with LA volumes [58]. These findings suggests that tissue-tracking on the LA wall can be a more sensitive marker to detect early myocardial disease among asymptomatic individuals despite the fact that LAEF might decrease, as documented in advanced decompensated cardiac disease (Fig. 6).

\section{Vascular imaging using CMR in MESA}

CMR has a distinct advantage over ultrasound in that three-dimensional visualization of the vessel wall and identification of components on the atherosclerotic plaque are possible. CMR has the unique ability to assess aortic geometry and function, representing vascular stiffness by vessel distensibility and pulse wave velocity with high reproducibility $[59,60]$.

Of over 3500 MESA participants with a mean age of 61 years, ascending aorta diameter was increased with age; higher aortic stiffness as measured by aortic distensibility was associated with hypertension, larger left ventricle, and incident CVD events (Fig. 7a) [60-63]. Individuals with high aortic stiffness had an almost 4-fold increase in risk of incident CVD compared to individuals with preserved aortic distensibility if they had a low-to-intermediate-risk CVD profile [62].

In a longitudinal MESA study over 10 years of followup, aortic stiffness was associated with wall thickness of the aorta, which increases by $0.032 \mathrm{~mm} /$ year [63]. Age, smoking, and increased blood pressure were associated with increases in aortic stiffness, but reduction in blood pressure was associated with less increase in the stiffness [64]. The loss of proximal aortic function was a robust

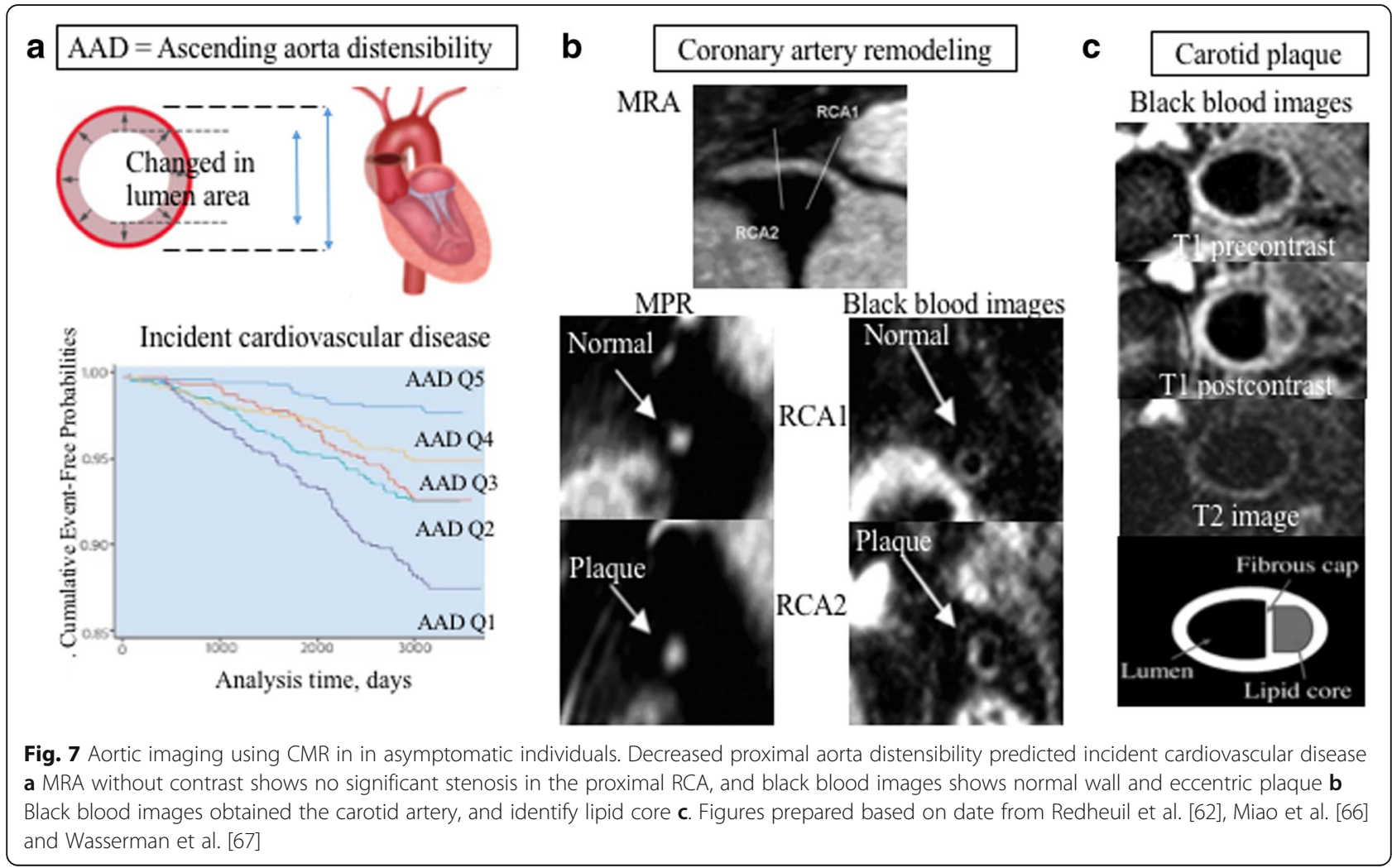


marker of vascular alteration, particularly in individuals with a lower traditional CVD risk profile as compared to those with a higher risk profile.

Atherosclerotic coronary artery plaque rupture is a key event leading to acute coronary syndromes. Black-blood coronary arterial wall CMR provides a unique new method to nonivasively image and assess the morphological features of the coronary arteries (Fig. 7b). MESA has reported the ability of CMR to identify coronary artery remodeling as an early marker of subclinical coronary atherosclerosis among participants with zero CAC score by computed tomography. The spatial resolution of CMR, however, needs improvement $[65,66]$.

MESA also has used black-blood sequences to noninvasively characterize carotid-artery atheroma composition (Fig. 7c). Using this technique, a lipid core was seen in $71 \%$ as selected from this asymptomatic population who had thickened carotid walls $(>1.5 \mathrm{~mm}$ by CMR), and plasma total cholesterol rather than other CVD risk factors, was strongly associated with lipid core presence by CMR [67]. In addition, carotid artery remodeling and lipid core presence were independent predictors of incident CVD events in MESA [68]. Consequently, vascular imaging features by CMR might be important predictors of cardiovascular risk in asymptomatic individuals.

\section{Conclusion}

MESA is one of the first large-scale population studies to use advanced CMR imaging techniques among asymptomatic individuals, and to follow up with repeated CMR examinations as part of a large multi-ethnic population study. A dominant pattern of LV remodeling across the life course is concentric remodeling by progressively reduced LV volumes. Progressively increased LV concentric remodeling is associated with interstitial fibrosis and is a marker of CVD events. Abnormal remodeling with LV hypertrophy is associated with replacement fibrosis, and a high risk for developing HF. Replacement scar, however, in more than half of the participants with scar by CMR LGE is undetected by ECG or by clinical adjudication.

Assessing LA and vascular geometry and function can be alternative tools to detect early stage subclinical disease. These CMR markers may significantly improve risk stratification in the general population. Finally, further advances in imaging are required to understand the role of risk factors and the progression of symptomatic disease pathways in the asymptomatic.

\section{Abbreviations}

CAC: Coronary artery calcium; CMR: Cardiovascular magnetic resonance; CVD: Cardiovascular diseases; HF: Heart failure; LA: Left atrial; LGE: Late gadolinium enhancement; LV:

Left ventricular; MESA: Multi-Ethnic Study of Atherosclerosis; MI: myocardial infarction; RV: Right ventricular

\section{Acknowledgements}

The authors thank the other investigators, staff, and participants of the MESA study for their valuable contributions. A full list of participating MESA investigators and institutions can be found at http://www.mesa-nhlbi.org.

\section{Funding}

This research was supported by contracts N01-HC-95159, N01-HC-95160, N01-HC-95161, N01-HC-95162, N01-HC-95163, N01-HC-95164, N01-HC-95165, N01-HC-95166, N01-HC-95167, N01-HC-95168, and N01-HC-95169 from the National Heart, Lung, and Blood Institute and by grants UL1-TR-000040 and UL1-TR-001079 from NCRR.

\section{Availability of data and materials \\ Not applicable.}

\section{Authors' contribution}

$\mathrm{KY}$, manuscript design, drafting, and revision, literature review, coordination among co-authors, final revisions. BAV, literature review, manuscript drafting and revision. DAB, literature review, manuscript drafting and revision. RLM, literature review, manuscript drafting and revision. $J L$, manuscript design, drafting, and revision, literature review, coordination among co-authors. All authors read and approved the manuscript.

Ethics approval and consent to participate

Not applicable.

\section{Consent for publication}

All authors declare their consent for publication.

\section{Competing interests}

The authors declare that they have no competing interests.

\section{Publisher's Note}

Springer Nature remains neutral with regard to jurisdictional claims in published maps and institutional affiliations.

\section{Author details}

'Department of Cardiology, Johns Hopkins University, Baltimore, MD, USA. ${ }^{2}$ Radiology and Imaging Sciences, National Institutes of Health Clinical Center, Bethesda, MD, USA. ${ }^{3}$ Department of Biostatistics, University of Washington, Seattle, WA, USA. ${ }^{4}$ St. Marianna University School of Medicine, Kawasaki, Japan. ${ }^{5}$ Professor of Medicine, Radiology and Epidemiology, Johns Hopkins Hospital, Johns Hopkins University, Blalock 524D1, 600 North Wolfe Street, Baltimore, MD 21287, USA.

Received: 22 January 2017 Accepted: 29 June 2017 Published online: 18 July 2017

References

1. Bild DE, Bluemke DA, Burke GL, Detrano R, Diez Roux AV, Folsom AR, et al. Multi-ethnic study of atherosclerosis: objectives and design. Am J Epidemiol. 2002:156:871-81.

2. Kawel N, Turkbey EB, Carr JJ, Eng J, Gomes AS, Hundley WG, et al. Normal left ventricular myocardial thickness for middle-aged and older subjects with steady-state free precession cardiac magnetic resonance: the multiethnic study of atherosclerosis. Circ Cardiovasc Imaging. 2012;5:500-8.

3. Natori S, Lai S, Finn JP, Gomes AS, Hundley WG, Jerosch-Herold M, et al. Cardiovascular function in multi-ethnic study of atherosclerosis: normal values by age, sex, and ethnicity. AJR Am J Roentgenol. 2006;186:S357-65.

4. Heckbert SR, Post W, Pearson GD, Arnett DK, Gomes AS, Jerosch-Herold M, et al. Traditional cardiovascular risk factors in relation to left ventricular mass, volume, and systolic function by cardiac magnetic resonance imaging: the multiethnic study of atherosclerosis. J Am Coll Cardiol. 2006;48:2285-92.

5. Cheng S, Fernandes VR, Bluemke DA, McClelland RL, Kronmal RA, Lima JA. Age-related left ventricular remodeling and associated risk for cardiovascular outcomes: the multi-ethnic study of atherosclerosis. Circ Cardiovasc Imaging. 2009;2:191-8.

6. Yoneyama K, Gjesdal O, Choi EY, Wu CO, Hundley WG, Gomes AS, et al. Age, sex, and hypertension-related remodeling influences left ventricular torsion assessed by tagged cardiac magnetic resonance in asymptomatic individuals: the multi-ethnic study of atherosclerosis. Circulation. 2012;126:2481-90. 
7. Roth GA, Forouzanfar MH, Moran AE, Barber R, Nguyen G, Feigin VL, et al. Demographic and epidemiologic drivers of global cardiovascular mortality. N Engl J Med. 2015;372:1333-41.

8. Eng J, McClelland RL, Gomes AS, Hundley WG, Cheng S, Wu CO, et al. Adverse Left Ventricular Remodeling and Age Assessed with Cardiac MR Imaging: The Multi-Ethnic Study of Atherosclerosis. Radiology. 2016:278:714-22.

9. Turkbey EB, McClelland RL, Kronmal RA, Burke GL, Bild DE, Tracy RP, et al. The impact of obesity on the left ventricle: the multi-ethnic study of atherosclerosis (MESA). JACC Cardiovasc Imaging. 2010;3:266-74.

10. Shah RV, Abbasi SA, Heydari B, Rickers C, Jacobs DR Jr, Wang L, et al. Insulin resistance, subclinical left ventricular remodeling, and the obesity paradox: MESA (multi-ethnic study of atherosclerosis). J Am Coll Cardiol. 2013;61:1698-706.

11. Shah RV, Murthy VL, Abbasi SA, Eng J, Wu C, Ouyang P, et al. Weight loss and progressive left ventricular remodelling: the multi-ethnic study of atherosclerosis (MESA). Eur J Prev Cardiol. 2015;22:1408-18.

12. Yoneyama K, Donekal S, Venkatesh BA, Wu CO, Liu CY, Souto Nacif M, et al. Natural history of myocardial function in an adult human population: serial longitudinal observations from MESA. JACC Cardiovasc Imaging. 2016;9:1164-73.

13. Van Hee VC, Adar SD, Szpiro AA, Barr RG, Bluemke DA, Diez Roux AV, et al, Exposure to traffic and left ventricular mass and function: the multi-ethnic study of atherosclerosis. Am J Respir Crit Care Med. 2009;179:827-34.

14. Ix JH, Katz R, Peralta CA, de Boer IH, Allison MA, Bluemke DA, et al. A high ankle brachial index is associated with greater left ventricular mass MESA (multi-ethnic study of atherosclerosis). J Am Coll Cardiol. 2010;55:342-9.

15. Van Hee VC, Adar SD, Szpiro AA, Barr RG, Diez Roux A, Bluemke DA, et al. Common genetic variation, residential proximity to traffic exposure, and left ventricular mass: the multi-ethnic study of atherosclerosis. Environ Health Perspect. 2010;118:962-9.

16. Turkbey EB, Jorgensen NW, Johnson WC, Bertoni AG, Polak JF, Diez Roux AV, et al. Physical activity and physiological cardiac remodelling in a community setting: the multi-ethnic study of atherosclerosis (MESA). Heart. 2010;96:42-8.

17. Heidenreich PA, Albert NM, Allen LA, Bluemke DA, Butler J, Fonarow GC, et al. Forecasting the impact of heart failure in the United States: a policy statement from the American Heart Association. Circ Heart Fail. 2013;6:606-19.

18. Jain A, Tandri H, Dalal D, Chahal H, Soliman EZ, Prineas RJ, et al. Diagnostic and prognostic utility of electrocardiography for left ventricular hypertrophy defined by magnetic resonance imaging in relationship to ethnicity: the multi-ethnic study of atherosclerosis (MESA). Am Heart J. 2010:159:652-8.

19. Armstrong AC, Gidding S, Gjesdal O, Wu C, Bluemke DA, Lima JA. LV mass assessed by echocardiography and CMR, cardiovascular outcomes, and medical practice. JACC Cardiovasc Imaging. 2012;5:837-48.

20. Jain A, McClelland RL, Polak JF, Shea S, Burke GL, Bild DE, et al. Cardiovascular imaging for assessing cardiovascular risk in asymptomatic men versus women: the multi-ethnic study of atherosclerosis (MESA). Circ Cardiovasc Imaging. 2011;4:8-15.

21. Bluemke DA, Kronmal RA, Lima JA, Liu K, Olson J, Burke GL, et al. The relationship of left ventricular mass and geometry to incident cardiovascular events: the MESA (multi-ethnic study of atherosclerosis) study. J Am Coll Cardiol. 2008;52:2148-55.

22. Chahal H, Bluemke DA, Wu CO, McClelland R, Liu K, Shea SJ, et al. Heart failure risk prediction in the multi-ethnic study of atherosclerosis. Heart. 2015;101:58-64.

23. Ambale-Venkatesh B, Yoneyama K, Sharma RK, Ohyama Y, Wu CO, Burke GL, et al. Left ventricular shape predicts different types of cardiovascular events in the general population. Heart. 2017;103:499-507.

24. Sibley CT, Noureldin RA, Gai N, Nacif MS, Liu S, Turkbey EB, et al. T1 mapping in cardiomyopathy at cardiac MR: comparison with endomyocardial biopsy. Radiology. 2012;265:724-32.

25. Ambale-Venkatesh B, Lima JA. Cardiac MRl: a central prognostic tool in myocardial fibrosis. Nat Rev Cardiol. 2015;12:18-29.

26. Wu KC, Weiss RG, Thiemann DR, Kitagawa K, Schmidt A, Dalal D, et al. Late gadolinium enhancement by cardiovascular magnetic resonance heralds an adverse prognosis in nonischemic cardiomyopathy. J Am Coll Cardiol. 2008;51:2414-21.

27. Briasoulis A, Mallikethi-Reddy S, Palla M, Alesh I, Afonso L. Myocardial fibrosis on cardiac magnetic resonance and cardiac outcomes in hypertrophic cardiomyopathy: a meta-analysis. Heart. 2015;101:1406-11.

28. Schelbert EB, Cao JJ, Sigurdsson S, Aspelund T, Kellman P, Aletras AH, et al. Prevalence and prognosis of unrecognized myocardial infarction determined by cardiac magnetic resonance in older adults. JAMA. 2012; 308:890-6.
29. Turkbey EB, Nacif MS, Guo M, McClelland RL, Teixeira PB, Bild DE, et al. Prevalence and correlates of myocardial scar in a US cohort. JAMA. 2015;314:1945-54

30. Masci PG, Schuurman R, Andrea B, Ripoli A, Coceani M, Chiappino S, et al. Myocardial fibrosis as a key determinant of left ventricular remodeling in idiopathic dilated Cardiomyopathy a contrast-enhanced cardiovascular magnetic study. Circulation-Cardiovascular Imaging. 2013; 6:790-9.

31. Ambale Venkatesh B, Volpe GJ, Donekal S, Mewton N, Liu CY, Shea S, et al. Association of longitudinal changes in left ventricular structure and function with myocardial fibrosis: the multi-ethnic study of atherosclerosis study. Hypertension. 2014;64:508-15.

32. Liu CY, Liu YC, Wu C, Armstrong A, Volpe GJ, van der Geest RJ, et al. Evaluation of age-related interstitial myocardial fibrosis with cardiac magnetic resonance contrast-enhanced T1 mapping: MESA (multi-ethnic study of atherosclerosis). J Am Coll Cardiol. 2013;62:1280-7.

33. Anversa P, Nadal-Ginard B. Myocyte renewal and ventricular remodelling. Nature. 2002;415:240-3.

34. Berk BC, Fujiwara K, Lehoux S. ECM remodeling in hypertensive heart disease. J Clin Invest. 2007;117:568-75.

35. Donekal S, Venkatesh BA, Liu YC, Liu CY, Yoneyama K, Wu CO, et al. Interstitial fibrosis, left ventricular remodeling, and myocardial mechanical behavior in a population-based multiethnic cohort: the multi-ethnic study of atherosclerosis (MESA) study. Circ Cardiovasc Imaging. 2014;7:292-302.

36. Yi CJ, Wu CO, Tee M, Liu CY, Volpe GJ, Prince MR, et al. The association between cardiovascular risk and cardiovascular magnetic resonance measures of fibrosis: the multi-ethnic study of atherosclerosis (MESA). J Cardiovasc Magn Reson. 2015;17:15.

37. Bursi F, Weston SA, Redfield MM, Jacobsen SJ, Pakhomov S, Nkomo VT, et al. Systolic and diastolic heart failure in the community. JAMA. 2006;296:2209-16.

38. Rosen BD, Lima JA, Nasir K, Edvardsen T, Folsom AR, Lai S, et al. Lower myocardial perfusion reserve is associated with decreased regional left ventricular function in asymptomatic participants of the multi-ethnic study of atherosclerosis. Circulation. 2006;114:289-97.

39. Edvardsen T, Rosen BD, Pan L, Jerosch-Herold M, Lai S, Hundley WG, et al. Regional diastolic dysfunction in individuals with left ventricular hypertrophy measured by tagged magnetic resonance imaging-the multiethnic study of atherosclerosis (MESA). Am Heart J. 2006;151:109-14.

40. Rosen BD, Edvardsen T, Lai S, Castillo E, Pan L, Jerosch-Herold M, et al. Left ventricular concentric remodeling is associated with decreased global and regional systolic function: the multi-ethnic study of atherosclerosis. Circulation. 2005;112:984-91.

41. Rosen BD, Saad MF, Shea S, Nasir K, Edvardsen T, Burke G, et al. Hypertension and smoking are associated with reduced regional left ventricular function in asymptomatic: individuals the multi-ethnic study of atherosclerosis. J Am Coll Cardiol. 2006;47:1150-8.

42. Fernandes VR, Polak JF, Edvardsen T, Carvalho B, Gomes A, Bluemke DA, et al. Subclinical atherosclerosis and incipient regional myocardial dysfunction in asymptomatic individuals: the multi-ethnic study of atherosclerosis (MESA). J Am Coll Cardiol. 2006;47:2420-8.

43. Almeida AL, Teixido-Tura G, Choi EY, Opdahl A, Fernandes VR, Wu CO, et al. Metabolic syndrome, strain, and reduced myocardial function: multi-ethnic study of atherosclerosis. Arq Bras Cardiol. 2014;102:327-35.

44. Opdahl A, Ambale Venkatesh B, Fernandes VR, Wu CO, Nasir K, Choi EY, et al. Resting heart rate as predictor for left ventricular dysfunction and heart failure: MESA (multi-ethnic study of atherosclerosis). J Am Coll Cardiol. 2014; 63:1182-9.

45. Choi EY, Rosen BD, Fernandes VR, Yan RT, Yoneyama K, Donekal S, et al. Prognostic value of myocardial circumferential strain for incident heart failure and cardiovascular events in asymptomatic individuals: the multiethnic study of atherosclerosis. Eur Heart J. 2013;34:2354-61.

46. Ambale-Venkatesh B, Armstrong AC, Liu CY, Donekal S, Yoneyama K, Wu $\mathrm{CO}$, et al. Diastolic function assessed from tagged MRI predicts heart failure and atrial fibrillation over an 8-year follow-up period: the multi-ethnic study of atherosclerosis. Eur Heart J Cardiovasc Imaging. 2014;15:442-9.

47. Kawel N, Nacif M, Arai AE, Gomes AS, Hundley WG, Johnson WC, et al. Trabeculated (noncompacted) and compact myocardium in adults: the multiethnic study of atherosclerosis. Circ Cardiovasc Imaging. 2012;5:357-66.

48. Captur G, Zemrak F, Muthurangu V, Petersen SE, Li C, Bassett P, et al. Fractal analysis of myocardial Trabeculations in 2547 study participants: multiethnic study of atherosclerosis. Radiology. 2015;277:707-15. 
49. Zemrak F, Ahlman MA, Captur G, Mohiddin SA, Kawel-Boehm N, Prince MR, et al. The relationship of left ventricular trabeculation to ventricular function and structure over a 9.5-year follow-up: the MESA study. J Am Coll Cardiol. 2014;64:1971-80.

50. Arbustini E, Weidemann F, Hall JL. Left ventricular noncompaction: a distinct cardiomyopathy or a trait shared by different cardiac diseases? J Am Coll Cardiol. 2014;64:1840-50.

51. Kawut SM, Lima JA, Barr RG, Chahal H, Jain A, Tandri $H$, et al. Sex and race differences in right ventricular structure and function: the multi-ethnic study of atherosclerosis-right ventricle study. Circulation. 2011;123:2542-51.

52. Leary PJ, Kaufman JD, Barr RG, Bluemke DA, Curl CL, Hough CL, et al. Trafficrelated air pollution and the right ventricle. The multi-ethnic study of atherosclerosis. Am J Respir Crit Care Med. 2014;189:1093-100.

53. Kawut SM, Barr RG, Lima JA, Praestgaard A, Johnson WC, Chahal H, et al. Right ventricular structure is associated with the risk of heart failure and cardiovascular death: the multi-ethnic study of atherosclerosis (MESA)-right ventricle study. Circulation. 2012;126:1681-8.

54. Vieira MJ, Teixeira R, Goncalves L, Gersh BJ. Left atrial mechanics: echocardiographic assessment and clinical implications. J Am Soc Echocardiogr. 2014;27:463-78.

55. Inoue YY, Alissa A, Khurram IM, Fukumoto K, Habibi M, Venkatesh BA, et al. Quantitative tissue-tracking cardiac magnetic resonance (CMR) of left atrial deformation and the risk of stroke in patients with atrial fibrillation. J Am Heart Assoc. 2015;4

56. Hoit BD. Left atrial size and function: role in prognosis. J Am Coll Cardiol. 2014;63:493-505.

57. Habibi M, Chahal H, Opdahl A, Gjesdal O, Helle-Valle TM, Heckbert SR, et al. Association of CMR-measured LA function with heart failure development: results from the MESA study. JACC Cardiovasc Imaging. 2014;7:570-9.

58. Imai M, Ambale Venkatesh B, Samiei S, Donekal S, Habibi M, Armstrong AC, et al. Multi-ethnic study of atherosclerosis: association between left atrial function using tissue tracking from cine MR imaging and myocardial fibrosis. Radiology. 2014;273:703-13.

59. Noda C, Ambale Venkatesh B, Ohyama Y, Liu CY, Chamera E, Redheuil A, et al. Reproducibility of functional aortic analysis using magnetic resonance imaging: the MESA. Eur Heart J Cardiovasc Imaging. 2016;17:909-17.

60. Turkbey EB, Jain A, Johnson C, Redheuil A, Arai AE, Gomes AS, et al. Determinants and normal values of ascending aortic diameter by age, gender, and race/ethnicity in the multi-ethnic study of atherosclerosis (MESA). J Magn Reson Imaging. 2014;39:360-8.

61. Al-Mallah MH, Nasir K, Katz R, Lima JA, Bluemke DA, Blumenthal RS, et al. Relation of thoracic aortic distensibility to left ventricular area (from the multi-ethnic study of atherosclerosis [MESA]). Am J Cardiol. 2014;113:178-82.

62. Redheuil A, Wu CO, Kachenoura N, Ohyama Y, Yan RT, Bertoni AG, et al. Proximal aortic distensibility is an independent predictor of all-cause mortality and incident CV events: the MESA study. J Am Coll Cardiol. 2014;64:2619-29.

63. Liu CY, Chen D, Bluemke DA, Wu CO, Teixido-Tura G, Chugh A, et al. Evolution of aortic wall thickness and stiffness with atherosclerosis: longterm follow up from the multi-ethnic study of atherosclerosis. Hypertension. 2015;65:1015-9.

64. Ohyama Y, Teixido-Tura G, Ambale-Venkatesh B, Noda C, Chugh AR, Liu CY, et al. Ten-year longitudinal change in aortic stiffness assessed by cardiac MRI in the second half of the human lifespan: the multi-ethnic study of atherosclerosis. Eur Heart J Cardiovasc Imaging. 2016;17:1044-53.

65. Macedo R, Chen S, Lai S, Shea S, Malayeri AA, Szklo M, et al. MRI detects increased coronary wall thickness in asymptomatic individuals: the multi-ethnic study of atherosclerosis (MESA). J Magn Reson Imaging. 2008;28:1108-15.

66. Miao C, Chen S, Macedo R, Lai S, Liu K, Li D, et al. Positive remodeling of the coronary arteries detected by magnetic resonance imaging in an asymptomatic population: MESA (multi-ethnic study of atherosclerosis). J Am Coll Cardiol. 2009;53:1708-15.

67. Wasserman BA, Sharrett AR, Lai S, Gomes AS, Cushman M, Folsom AR, et al. Risk factor associations with the presence of a lipid core in carotid plaque of asymptomatic individuals using high-resolution MRI: the multi-ethnic study of atherosclerosis (MESA). Stroke. 2008;39:329-35.

68. Zavodni AE, Wasserman BA, McClelland RL, Gomes AS, Folsom AR, Polak JF, et al. Carotid artery plaque morphology and composition in relation to incident cardiovascular events: the multi-ethnic study of atherosclerosis (MESA). Radiology. 2014;271:381-9.

69. Ebong IA, Watson KE, Goff DC Jr, Bluemke DA, Srikanthan P, Horwich T, et al. Age at menopause and incident heart failure: the multi-ethnic study of atherosclerosis. Menopause. 2014;21:585-91.

\section{Submit your next manuscript to BioMed Central and we will help you at every step:}

- We accept pre-submission inquiries

- Our selector tool helps you to find the most relevant journal

- We provide round the clock customer support

- Convenient online submission

- Thorough peer review

- Inclusion in PubMed and all major indexing services

- Maximum visibility for your research

Submit your manuscript at www.biomedcentral.com/submit 